\title{
Gestational choriocarcinoma presenting as endobronchial carcinoma
}

\author{
D T MCLEOD
}

From the Department of Medicine, Godfrey Huggins School of Medicine, Harare, Zimbabwe

ABSTRACT A patient is reported who presented with features of bronchial obstruction due to endobronchial tumour. Failure to recognise this as being due to choriocarcinoma led to a delay in diagnosis and effective treatment which, given earlier, may have proved lifesaving.

Choriocarcinoma usually occurs in young women and grows rapidly with widespread haematogenous dissemination. It is a potentially curable malignancy owing to its responsiveness to chemotherapy. With early diagnosis and vigorous treatment remission rates of $70 \%$ are reported in patients with metastatic disease'; with cerebral metastases the remission rate falls to $50 \%$.' Thus awareness of the various modes of presentation and avoidance of delay in diagnosis greatly improves prognosis.

\section{Case history}

A 21 year old woman was referred with a three month history of increasing exertional dyspnoea, central chest pain, and a dry cough occasionally accompanied by a little haemoptysis. Copious vomiting and weight loss had occurred over the preceding month. She had consulted a gynaecologist because of amenorrhoea and lower abdominal pain, which culminated in a bloody vaginal discharge. She was considered to be menstruating but when reviewed a month later she still had vaginal bleeding. Examination showed a bulky uterus with an open cervical os; a threatened abortion was suspected but abdominal ultrasound showed a non-gravid uterus. She was therefore treated as an outpatient with oral tetracycline 500 $\mathrm{mg}$ four times daily and oral metronidazole $\mathbf{4 0 0} \mathrm{mg}$ thrice daily to deal with any pelvic sepsis. Her previous menstrual history was unremarkable and her only pregnancy had resulted in a live birth three years previously.

Clinical examination showed a considerably wasted young woman. There was no clubbing, jaundice, or lymphadenopathy. Her temperature was $37.5^{\circ} \mathrm{C}$ and pulse rate 90 beats/ min regular, and her jugular venous pressure was not raised. Her blood pressure was $100 / 70 \mathrm{~mm} \mathrm{Hg}$ and heart sounds were normal, and there was no peripheral oedema. Signs of a left sided pleural effusion were noted but the striking finding was absence of breath sounds anteriorly over the left upper lobe. There were no abdominal masses. Apart from mild

Address for reprint requests: Dr D T McLeod, Department of Respiratory Medicine, Freeman Hospital, Newcastle Upon Tyne NE7 7DN.

Accepted 26 January 1988 confusion examination of the central nervous system showed $\overrightarrow{\vec{\omega}}$ nothing abnormal.

The initial haemoglobin concentration was $10.4 \mathrm{~g} / \mathrm{dl}$, white cell count $12.4 \times 10^{9} / 1$ with $75 \%$ neutrophils, erythrocyte $\stackrel{\vec{x}}{.}$ sedimentation rate $124 \mathrm{~mm}$ in the first hour (Westergren), $\vec{\omega}$ plasma sodium concentration $133 \mathrm{mmol} / 1$, potassium $4.1 \mathrm{cr}$ $\mathrm{mmol} / \mathrm{l}$, and urea $5.0 \mathrm{mmol} / 1$. A chest radiograph showed a $A$ mass in the left mid zone and a pleural effusion (figure). Fluid $\vec{\circ}$ could not be obtained from the pleural space. At broncho- 음 scopy a necrotic, red tumour mass was seen occluding the left $\rightarrow$ upper lobe bronchus; all other bronchi were normal. His- $z$ tological examination of the biopsy material showed large anaplastic malignant cells. She was referred to a thoracic surgeon for a repeat bronchoscopy and possible ${ }_{\infty}$ thoracotomy. The patient, however, decided to spend three $\infty$ weeks at Christmas with her family. On her return her $\square$ condition had worsened and a laboratory report suggested acid alcohol fast bacilli in her sputum, so she was transferred to an infectious diseases hospital. The result of a Mantoux test (1:1000) was negative. A chest radiograph now showed complete opacification of the left hemithorax with medias- $\varrho$ tinal shift to the right. As no further acid alcohol fast bacilli $\overrightarrow{\vec{\sigma}}$ were found in seven early morning sputum samples fibreoptic $\frac{0}{3}$ bronchoscopy was repeated. Tumour was invading and $\vec{\supset}$ occluding the left lower lobe as well as the left upper lobe bronchi. Malignant cells were confirmed in the biopsy material. A pleural tap produced blood stained fluid and she $\overrightarrow{0}$ was considered to be inoperable.

Review of the histology with the pathologists did not $x$ identify the tumour. In view of her age a pregnancy test was $\frac{}{3}$ performed and the result was positive, as it had been twice. three months earlier, though this had been overlooked. A $24 \%$

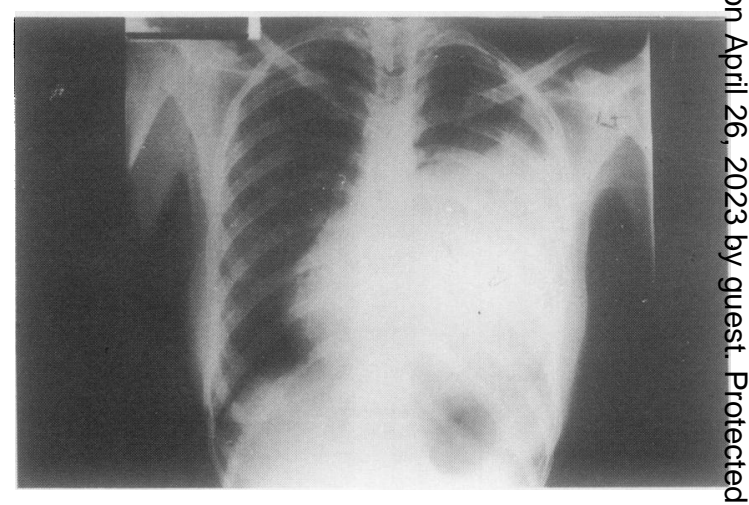

Chest radiograph at presentation showing a mass in the left midzone and a pleural effusion. 
hour urine human chorionic gonadotrophin concentration was $1421400 \mathrm{IU}$ (normal $400 \mathrm{IU} / \mathrm{l}$ in a pregnant woman). The $\beta$ human chorionic gonadotrophin subunit concentration was $2258 \mathrm{IU} / \mathrm{ml}$ in the serum and $2300 \mathrm{IU} / \mathrm{ml}$ in the cerebrospinal fluid, which confirmed choriocarcinoma with probable cerebral metastases. Abdominal ultrasound showed a normal liver and a normal sized non-gravid uterus; there was no trophoblastic tissue.

After the first of two grand mal convulsions an electroencephalogram (EEG) showed a mild, diffuse, slow abnormal trace with no evidence of a focal lesion. Computed tomography of the head was not available. As the clinical picture put her in the "poor prognosis" category she was given cytotoxic chemotherapy with intravenous actinomycin D $0.5 \mathrm{mg}$ and methotrexate $25 \mathrm{mg}$, and in addition intrathecal methotrexate $12.5 \mathrm{mg}$, intravenous cyclophosphamide $500 \mathrm{mg}$, and intravenous vincristine $1 \mathrm{mg}$.

Although she improved clinically bone marrow suppression occurred (haemoglobin $8.8 \mathrm{~g} / \mathrm{dl}$, white cell count $2.3 \times$ $10^{4} / 1$, and platelets $\left.28 \times 10^{9} / 1\right)$, necessitating a delay of one month before the second pulse could be given. Five days after starting her second pulse she deteriorated and died. Permission for a necropsy was not granted.

\section{Discussion}

Respiratory symptoms are a common mode of presentation of choriocarcinoma. In a series of 24 patients three presented with haemoptysis and two with cough and dyspnoea. ${ }^{2}$ Haemoptysis is thought to arise from alveolar haemorrhage around metastatic nodules, though in this patient haemoptysis was the result of endobronchial tumour. Isolated pulmonary metastases suggesting primary pulmonary carcinoma may occur, though this is rare in Africa. ${ }^{3}$ This patient had a mass in the left mid zone, the right lung was clear, and she went on to develop a bloody pleural effusion. Pleural effusion is usually blood stained and results from the rupture of a superficial pulmonary nodule with bleeding into the pleural space, ${ }^{4}$ though haemopneumothorax may result.

Confusion, grand mal convulsions, and a serum:cerebrospinal fluid ratio of $\beta$ subunit human chorionic gonadotro- phin of less than 60 indicate cerebral metastases and poorer prognosis. Kobayashi et $a^{5}$ in an necropsy proved series found an overall incidence of 24 in 87 patients, with single lesions in two thirds. The mechanism of tumour spread is probably arterial embolisation, an event that may take place without obvious pulmonary lesions through arteriovenous shunting in the lungs. The most frequently used diagnostic test for brain metastases is a technetium-99m brain scan and where necessary a computed tomography scan of the head. Unfortunately neither test was available for this patient. Electroencephalography has been found to be unhelpful.

Pulmonary metastases do not appear to affect prognosis adversely and after successful chemotherapy with a return of human chorionic gonatrophin concentrations to normal pulmonary metastases may persist. Resected nodules on histological examination show only fibrosis with central cellular debris but no evidence of viable tumour. ${ }^{46}$ Brain metastasis, on the other hand, is a bad prognostic feature, though a survival rate of $50 \%$ is possible with aggressive treatment.

This young woman presented with endobronchial choriocarcinoma, an unusual pulmonary presentation, which was misdiagnosed and so effective treatment was delayed. This report highlights an unusual presentation of choriocarcinoma in the hope that in the future others will benefit.

\section{References}

1 Weed JC, Hammond CB. Cerebral metastatic choriocarcinoma: Intensive therapy and prognosis. Obstet Gynecol 1980;55:89-94.

2 Davey DA, Fray R. Choriocarcinoma and invasive mole: a review of 10 years experience. SA Med J 1979;56:924-31.

3 Evans KT, Cockshott WP, Hendrickse JP. Pulmonary changes in malignant trophoblastic disease. Br J Radiol 1965;38:161-71.

4 Tow SH. The pulmonary lesion in choriocarcinoma. Proc $R$ Soc Med 1967;60:239-40.

5 Kobayashi T, Kida Y, Yoshida J, Shibuya N, Kageyama N. Brain metastasis of choriocarcinoma. Surg Neurol 1982;17:395-403.

6 Swett HA, Westcott JI. Residual non malignant pulmonary nodules in choriocarcinoma. Chest 1974;65:560-2. 